\title{
Compliance in amblyopia therapy: objective monitoring of occlusion
}

\author{
Alistair R Fielder, Mary Irwin, Rosemary Auld, Kenneth D Cocker, Helen S Jones, \\ Merrick J Moseley
}

\begin{abstract}
Aim/Background-This study aimed to determine the feasibility of objective compliance monitoring of amblyopia therapy in clinical research. Occlusion has been the mainstay of amblyopia therapy for over 250 years, yet it has never been subjected to rigorous evaluation. Treatment regimens range arbitrarily from a few minutes to most of the waking hours of the day. Compliance is problematic and as, hitherto, accurate objective monitoring has been impossible it is not known how much occlusion is required to effect an improvement in vision.
\end{abstract}

Methods-An occlusion dose monitor (ODM) has been developed. The ODM consists of a modified occlusion patch and a miniature battery driven datalogger which periodically monitors patch skin contact. The patch is a standard disposable item with two miniature electrocardiogram electrodes attached to its undersurface. The datalogger comprises a high speed static RAM and a clock driven address counter. Data are retrieved using an IBM PC/AT computer. Fifteen child amblyopes were randomly allocated unilateral occlusion of 1,4 , or 8 hours per day for 4 weeks. Owing to data loss, presumed because of accumulation and discharge of static electricity, an additional child was included in the 8 hour group. Outcome measures were objective (ODM) and subjective (diary) compliance with treatment, $\log M A R$ visual acuity, and contrast sensitivity.

Results-Objective monitoring of occlusion is technically feasible and clinically informative.

Conclusion-Objective monitoring of occlusion has opened up new research opportunities which, it is hoped, will enable the dose-effect relation of occlusion therapy in the various types of amblyopia to be investigated objectively, and facilitate the design of effective therapeutic regimens.

(Brf Ophthalmol 1995; 79: 585-589)

Amblyopia is the commonest vision defect of childhood, with a prevalence of about $1 \%$ to $5 \% .{ }^{1}$ Over the past few decades it has attracted tremendous interest with neuroscientific investigations, exemplified by the work of the Nobel prize winners Hubel and Weisel who demonstrated that the developing visual system is exquisitely sensitive to deprivation. This led to the concept of a sensitive period for amblyopia, its genesis and treatment, and the recognition that this defect must be identified in early childhood if treatment is to be successful. The impact of this fundamental research on health service delivery has been dramatic with the establishment of vision screening programmes, both preschool in at least 160 of 203 UK health authorities, ${ }^{2}$ and again on school entry. Thus, by 5 or 6 years of age the vast majority of the 500000 or so annual UK school entrants have been screened for amblyopia two, three, or more times. ${ }^{2}$

With such a massive investment of research and clinical resources it behoves us to ensure that amblyopia therapy is effective. Introduced over 250 years ago, occlusion remains even today the mainstay of treatment with reported success rates ranging from $30 \%{ }^{3}$ to $93 \% .^{45}$ Even 'success' needs defining as a recent population based study ${ }^{6}$ found that only $60 \%$ of children achieved acuities of $6 / 12$ or better, and of those with very poor acuity $(\leqslant 6 / 60)$ only $13 \%$ reached $6 / 18$.

In 1973 Duke-Elder ${ }^{7}$ pronounced that 'occlusion should be total and continuous ... the use of an intermittent form ... is an illusory and valueless procedure' a sentiment echoed 17 years later by von Noorden who recommended that occlusion should be worn 'completely and constantly during all waking hours'. ${ }^{1}$ Contrary to this advice, lesser amounts of occlusion are in everyday use, even for as little as a few minutes a day. ${ }^{8}$ These diverse regimens serve only to highlight that the amount of occlusion necessary to produce an improvement of vision is quite unknown and at a clinical level this has resulted in the production of the simple pragmatically derived occlusion protocols found in paediatric ophthalmic texts. 910

For many medical treatment regimens compliance is a critical issue and reviews of this literature quote an overall non-compliance rate of around 50\%. ${ }^{11}$ Amblyopia therapy is not exempt from the problem of poor compliance; indeed, occlusion as a mode of treatment itself has been identified as one of the major determinants of poor outcome. ${ }^{12-14}$ This is not at all surprising because occlusion has many features favouring non-compliance such as patient unacceptability (for example, forced to use the worse eye, occlusor on face, cosmetic issues), parental inconvenience, and lengthy treatment duration. Thus in two crude qualitative compliance studies, ${ }^{1214}$ low initial acuity has been reported to be associated with poor compliance which reduced the chance of a favourable outcome. To increase the amount 


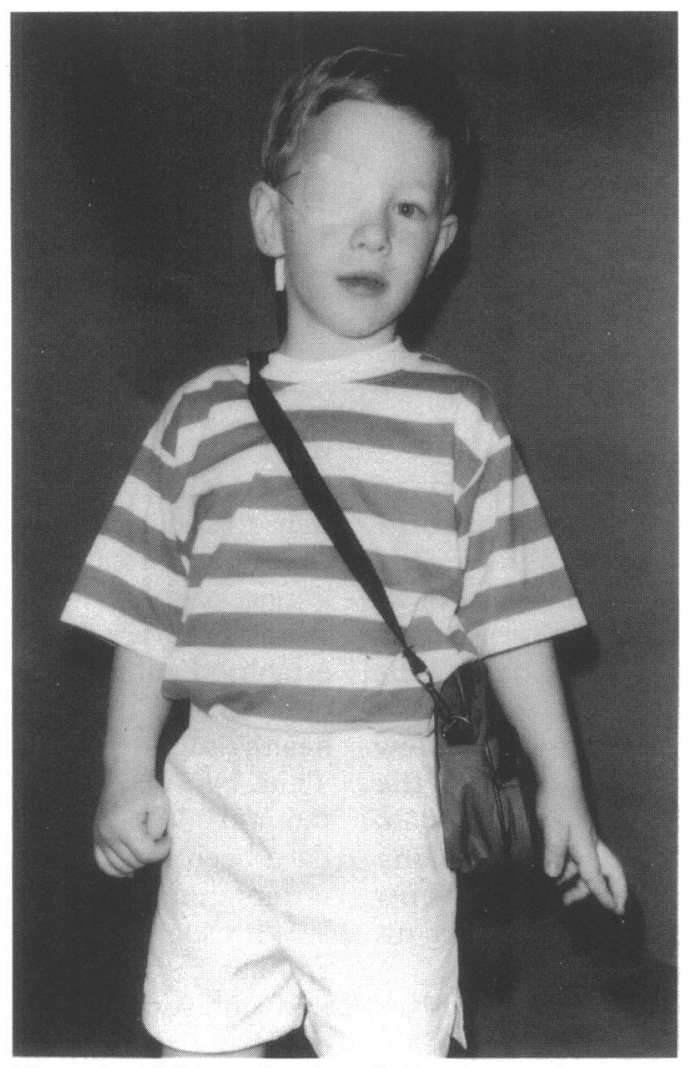

Figure 1 Child wearing the occlusion dose monitor.

of time the occlusor is worn by a child a range of methods is employed including encouraging, ordering, pleading, threatening or bribery, ${ }^{59}$ and sometimes hospital admission. ${ }^{15}$ The extreme methods of arm restraint by a plaster cast $^{5}$ and eyelid suturing ${ }^{16}$ must be confined to the past. While the above might all improve compliance as defined by the Oxford English Dictionary - 'action in accordance with request or command' they are not embodied in a more user friendly definition 'patient acceptance of recommended health behaviours'. ${ }^{11}$

As we have not so far been able objectively to monitor compliance we are a long way from being able 'to assess completely the impact of various occlusion regimens on long-term gains in visual performance'. ${ }^{17}$ In other words we have no understanding of the dose-effect relation of occlusion in amblyopia therapy. To determine treatment effectiveness, we require accurate dosage information: the daily regimen (dose rate) and accumulated dose (dose), which are dependent upon accurate compliance data being available. In general, there are three methods to evaluate compliance: listening to the patient, counting 'pills', and assessing a drug metabolite or other marker. ${ }^{11}$ When applied to amblyopia therapy, the first of these is clearly not applicable in childhood, and the closest one can approach the second is the keeping of an occlusion diary by the parent. ${ }^{14}$ However, not every 'pill' prescribed enters the body, and similarly not every occlusion patch is worn for the specified period, and while one can count patches, that may be no indication of the time worn. While a diary is an attempt to monitor occlusion it has a few fundamental weaknesses: it is not free of bias, its completion is likely to be influenced by the monitoring process, ${ }^{18}$ and it requires observation of the entire treatment period which is impossible in almost every case. In amblyopia therapy the analogue of measuring a metabolite for drug compliance is the monitoring of visual functions. However, as such outcome measures presuppose precise knowledge of the dose administered they are not appropriate. Thus for a number of reasons, none of these methods is suitable to monitor occlusion therapy accurately and objectively.

Smith refers to the 'poverty of medical evidence' ${ }^{19}$ in describing treatments which are accepted as clinical dogma but have never been subjected to rigorous evaluation. Occlusion therapy for amblyopia falls into this category owing to our paucity of knowledge on the dose-effect relation - a situation one finds hard to imagine for any comparably established therapy outside ophthalmology.

In this paper we describe pilot work using a recently developed occlusion dose monitor (ODM) which enables, for the first time, objective monitoring of occlusion therapy. ${ }^{20}$ Our aim was to determine the feasibility of objective monitoring of occlusion therapy using the ODM in clinical research. Visual performance data are included solely to demonstrate how clinical outcomes can be misinterpreted in the absence of knowledge of compliance. The dose-effect relation, which may be affected by age, amblyopia type, initial acuity, etc, will be the subject of future research.

\section{Patients and methods}

The occlusion dose monitor (ODM) consists of a modified occlusion patch and a miniature battery driven datalogger (Fig 1). The patch is a standard, commercially available, disposable item, with two miniature electrocardiogram electrodes attached to its undersurface. Occlusor-skin contact is inferred from reduced resistance across the patch electrodes, sampled every 64 seconds. Leads, easily concealed under clothing, connect the patch to the datalogger (weight $150 \mathrm{~g}$ and dimensions $115 \times 70 \times 30 \mathrm{~mm}$ ) 'Walkman-style' which is carried in a shoulder bag. The datalogger comprises a high speed static RAM and a clock driven address counter. There are no patient operated controls. On return to the clinic, the ODM is interrogated by an IBM PC/AT computer (a process taking less than 10 minutes) and the occlusion time-history stored for subsequent analysis.

Parents were given the modified patches and instructed how to connect the leads to the datalogger which could be plugged in either way ignoring polarity. After each occlusion period the patch was peeled off and the leads disconnected. A new patch was used for each occlusion period.

The pilot study was directed entirely towards the evaluation of the ODM within a clinical research setting and was based on the single case research design. ${ }^{21}$ Permission was obtained from the ethics committee of the 
Table 1 Details of data loss by accumulation of static electricity within the ODM. The child who withdrew from the study, and the additional illustrative case 3 are not included

\begin{tabular}{lllll}
\hline $\begin{array}{l}\text { Subject } \\
\text { number }\end{array}$ & $\begin{array}{l}\text { Prescribed } \\
\text { (hours } \\
\text { per day) }\end{array}$ & $\begin{array}{l}\text { Treatment } \\
\text { duration } \\
\text { (days) }\end{array}$ & $\begin{array}{l}\text { Days } \\
\text { containing } \\
\text { data loss }\end{array}$ & $\begin{array}{l}\text { Proportion } \\
\text { of data } \\
\text { loss (\%) }\end{array}$ \\
\hline 1 & 1 & 29 & 3 & 10 \\
2 & 1 & 28 & 0 & 0 \\
$3^{\star}$ & 1 & 29 & 0 & 0 \\
$4^{\star \star}$ & 1 & 28 & 2 & 7 \\
5 & 4 & 32 & 27 & 84 \\
6 & 4 & 29 & 13 & 45 \\
7 & 4 & 29 & 7 & 24 \\
8 & 4 & 43 & 14 & 33 \\
9 & 4 & 28 & 0 & 0 \\
10 & 8 & 29 & 7 & 24 \\
11 & 8 & 38 & 3 & 8 \\
12 & 8 & 30 & 17 & 57 \\
13 & 8 & 31 & 13 & 42 \\
14 & 8 & 32 & 25 & 78 \\
\hline
\end{tabular}

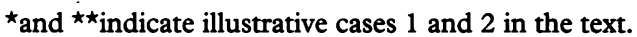

West Birmingham Health Authority, and parents gave informed consent before their children entered the study. Subjects were recruited using the following inclusion criteria: children presenting with untreated amblyopia, an ability to perform a (logMAR) linear visual acuity test ${ }^{22}$ and the Pelli-Robson contrast sensitivity test, and a willingness to attend for review on a weekly basis for the trial period. The study consisted of three phases: assessment, baseline, and treatment. In the assessment phase, children underwent a full ophthalmic (including cycloplegic refraction) and orthoptic examination. Spectacles were prescribed if necessary. After a minimum of 14 days of adaptive spectacle wear, or immediately if spectacles were not prescribed, subjects entered the baseline phase in which visual performance was measured on two occasions, 1 week apart. This provided a quantitative indication of the combined effects of test reliability, practice (perceptual learning), and adaptation to spectacle wear. Treatment began concurrently with the end of the baseline phase and children were randomly prescribed minimal ( 1 hour per day), part time ( 4 hours per day), or full time ( 8 hours per day) occlusion.

Parents were instructed in the use of the ODM and were also provided with a diary to record the start and finish of all periods of occlusion. Treatment duration was 4 weeks with subjects returning at weekly intervals for visual performance assessment, and subjective (diary) and objective (ODM) occlusion data retrieval. Standard clinical care was resumed at the end of treatment.

One additional patient was recruited from a later study of children with complex ophthalmic problems of which amblyopia was but one component (but who otherwise met the inclusion criteria of the pilot study). For reasons to be explained later, the ODM had by this time been modified and utilised a commercially available datalogger ('Tinytalk', Orion Components Chichester Ltd, UK) with a revised sample interval of 3.2 minutes. Outcome measures were: distance visual acuity, contrast sensitivity, compliance, and patient and parental impressions of ODM use. To quantify compliance we formulated three indices:
(1) Monitor compliance ratio $(\mathrm{MCR})=$ occlusion recorded by the ODM/prescribed occlusion.

(2) Diary compliance ratio $(\mathrm{DCR})=$ occlusion recorded in the diary/prescribed occlusion.

(3) Precision index $(\mathrm{PI})=\mathrm{MCR} / \mathrm{DCR}-$ that is, agreement between objective and subjective records.

\section{Results}

Fourteen children have so far completed our protocol for ODM evaluation. Without exception the device has proved acceptable to all children and their parents. There were no complaints specific to the use of the ODM as opposed to occlusion itself ( $t$ and Table 1$)$. Three children developed skin allergies caused by the adhesive occlusor, which were relieved by changing to a non-allergenic modified patch. One child fell off his bike while undergoing occlusion and dislodged the components within the ODM. Generally, parents completed the occlusion diary satisfactorily, with only a few gaps, although in some entries handwriting was difficult to decipher. Where ODM and diary data were available they generally correlated well, as shown in the illustrative case studies below. There were some exceptions, and in these cases, diary data were often better matched to the prescribed regimen than to the ODM data, with instances of both under and overrecording of occlusion in the diary. In addition, there were a few occasions when a continuous occlusion period in the diary corresponded to a period of ODM data containing several short breaks where intermittent occlusor-skin contact may have occurred.

\section{ILLUSTRATIVE CASE STUDIES}

Unlike more familiar methods, the single case research design is essentially the application of rigorous quantitative methods (in terms of treatment and outcome variables) to the traditional case history. Having demonstrated the feasibility of ODM use, we present three cases to emphasise the benefits that this new technique may bring to clinical practice.

\section{Subject 1 (pilot study)}

A 4-year-old girl with anisometropic amblyopia of the right eye was prescribed 1 hour of occlusion per day. Full clinic attendance was attained and only on 2 out of 28 days' occlusion was there no attempt made to patch the child's eye. The total prescribed dose was 1680 minutes, with objective (ODM) and subjective (diary) recordings of 1605 and 1563 minutes

†We did, however, initially encounter technical difficulties of data loss resulting from previously stored ODM data being overwritten. This was presumed to be due to the accumulation and discharge of static electricity within the ODM and especially prone to occur with long periods of occlusion. To overcome this problem ODM construction was modified as described earlier to include a commercially available datalogger which has subsequently achieved a degree of reliability accept- 

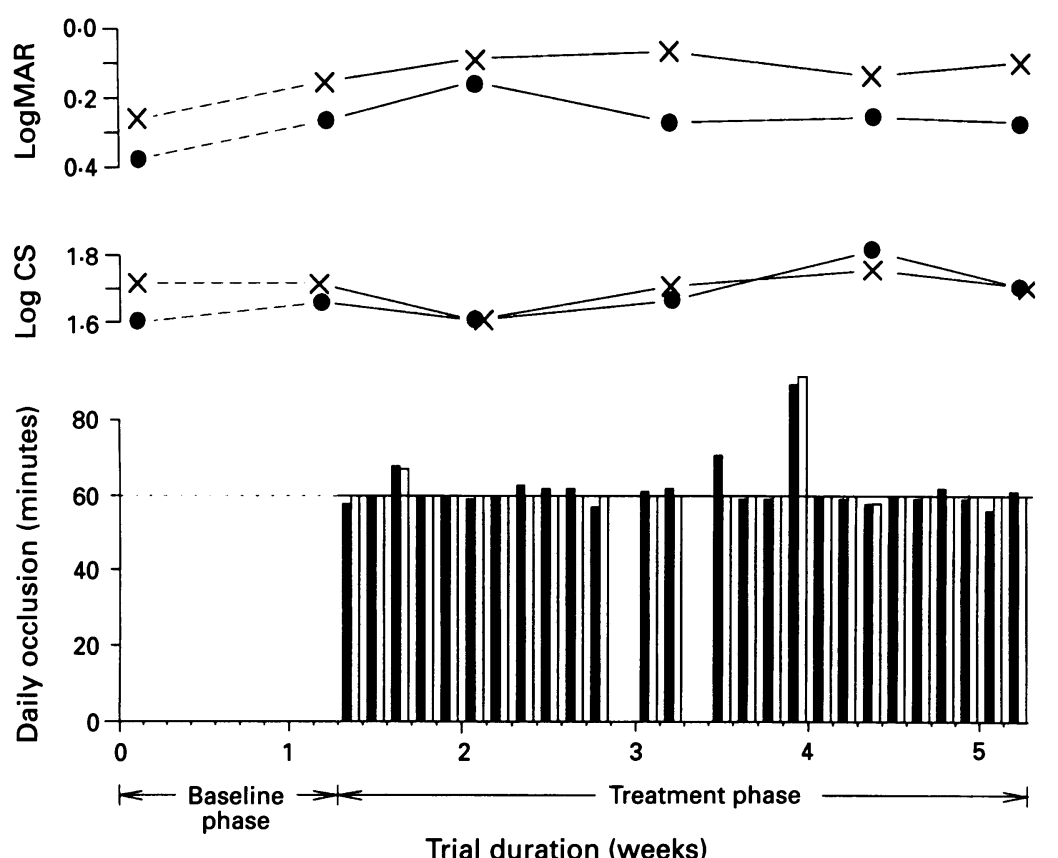

Figure 2 Given the excellent objectively determined compliance we can rule out non-compliance as an explanation for poor outcome (subject 1). Two upper line graphs show visual acuity and contrast sensitivity as a function of trial duration; (O) amblyopic eye; (X) fellow eye. Broken lines indicate visual function during baseline phase. Lower bar graph shows daily occlusion recorded objectively (ODM, $\square$ ) and subjectively (diary, $\square$ ). Horizontal line indicates the prescribed occlusion dose (..... $)$.

respectively yielding the following compliance indices: $\quad \mathrm{MCR}=0.96, \quad \mathrm{DCR}=0.93$, and $\mathrm{PI}=1 \cdot 03$. Compliance, as a function of treatment duration and the corresponding change in visual performance, is shown in Figure 2. Despite a small unpredicted improvement in acuity in the fellow eye $(0.11 \mathrm{log}$ units), compared with the mean baseline, the improvement in either acuity $(0.05 \mathrm{log}$ units) or contrast sensitivity in the amblyopic eye (0.08 log units) was not clinically significant.
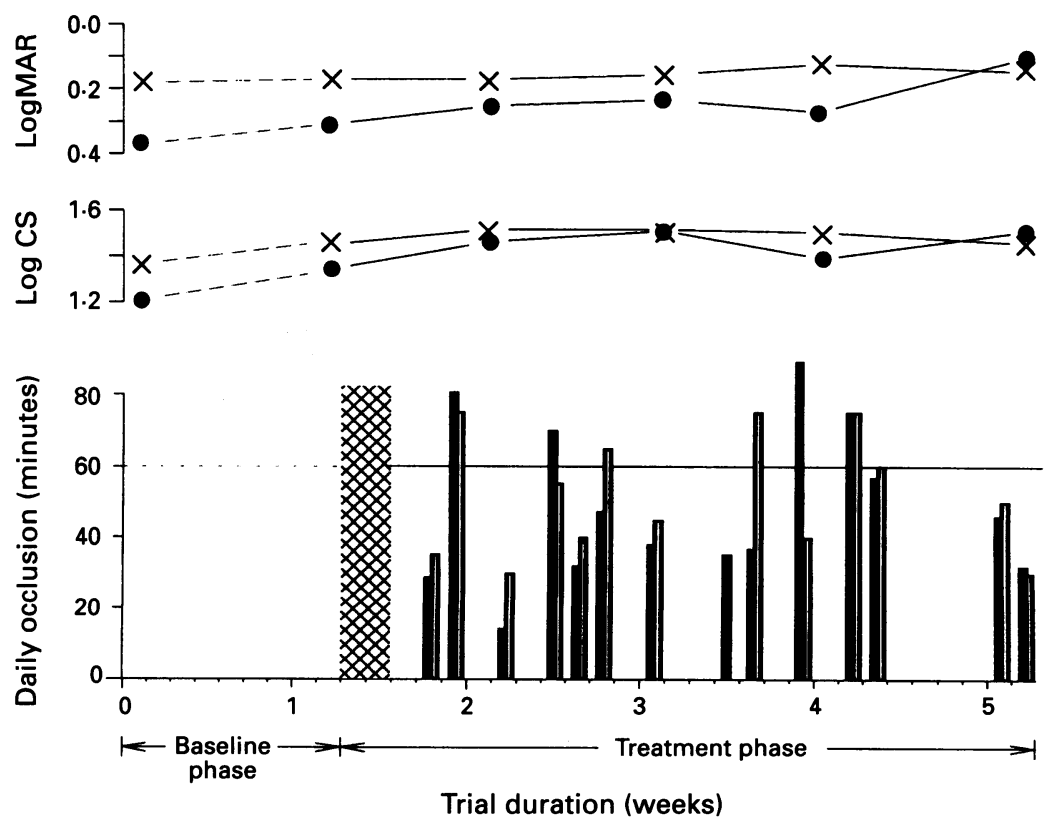

Figure 3 Compliance is generally only questioned in the case of outcome failure and success in this case would have been incorrectly attributed to strict adherence to the prescribed regimen (subject 2). Hatched area on lower bar graph indicates days loss of data. Key as for Figure 1.
Subject 2 (pilot study)

This 4-year-old boy had anisometropic amblyopia of his left eye, was prescribed spectacles and, after adaptation, allocated 1 hour of occlusion per day. The total prescribed dose was 1560 minutes, with ODM record of 624 minutes and diary record of 675 minutes respectively: $\mathrm{MCR}=0.40$, $\mathrm{DCR}=0.43, \mathrm{PI}=0.93$. In this case, compliance was poor, but although occlusion was worn only $40 \%$ of the prescribed time, acuity improved from mean baseline $0.34 \log$ MAR to $0.1 \log$ MAR in the amblyopic eye (Fig 3). A concomitant improvement in contrast sensitivity (0.23 log units) was also recorded.

\section{Subject 3 (modified ODM)}

This male child, born at 26 weeks' gestation, developed early stage 3 retinopathy of prematurity in each eye, which resolved, and then at 3 years presented with a left convergent strabismus.

There was no posterior pole retinal abnormality (major vessel angle $110^{\circ}$ right and left eyes). Refraction revealed +0.75 DS in the right and emmetropia in the left eye. He was prescribed 8 hours of occlusion per day, 13440 minutes in total, and achieved ODM and diary recordings of 8976 and 9429 minutes. Compliance indices: $\mathrm{MCR}=0.67$, $\mathrm{DCR}=0.70$, and $\mathrm{PI}=0.95$ (Fig 4). Acuity in the amblyopic eye improved by about 6 lines during the baseline phase $(0.9$ to 0.34 $\log$ units) and three additional letters with occlusion; note also the improvement in contrast sensitivity $(0.43 \mathrm{log}$ units from mean baseline). This subject demonstrates that even lengthy periods of occlusion can be monitored objectively.

\section{Discussion}

Occlusion therapy can now be monitored using an occlusion dose monitor (ODM). At this early stage we are not concerned with the effectiveness of monitored occlusion on amblyopia, but simply with validating objective compliance monitoring in the 'field' - that is, its feasibility for future clinical research. We are aware of potential pitfalls, such as failure to connect the leads to the ODM, or placing the occlusor on an arm, for instance, rather than over the eye. While the latter remains a possibility, for a child to repeatedly wear a falsely located occlusor for up to 8 hours per day for 4 weeks requires a vivid imagination and a devious tenacity of unlikely proportions. Notwithstanding these concerns, we have shown that the amount of occlusion that a child receives between visits can now be monitored objectively using an ODM, even for long occlusion periods (subject 3 ). It has proved acceptable to patients and their parents, and no patient related issues have been encountered which might pose major problems for its future clinical use, at this age and in younger children.

In our study we would expect the parental diary to correlate well with ODM data as 

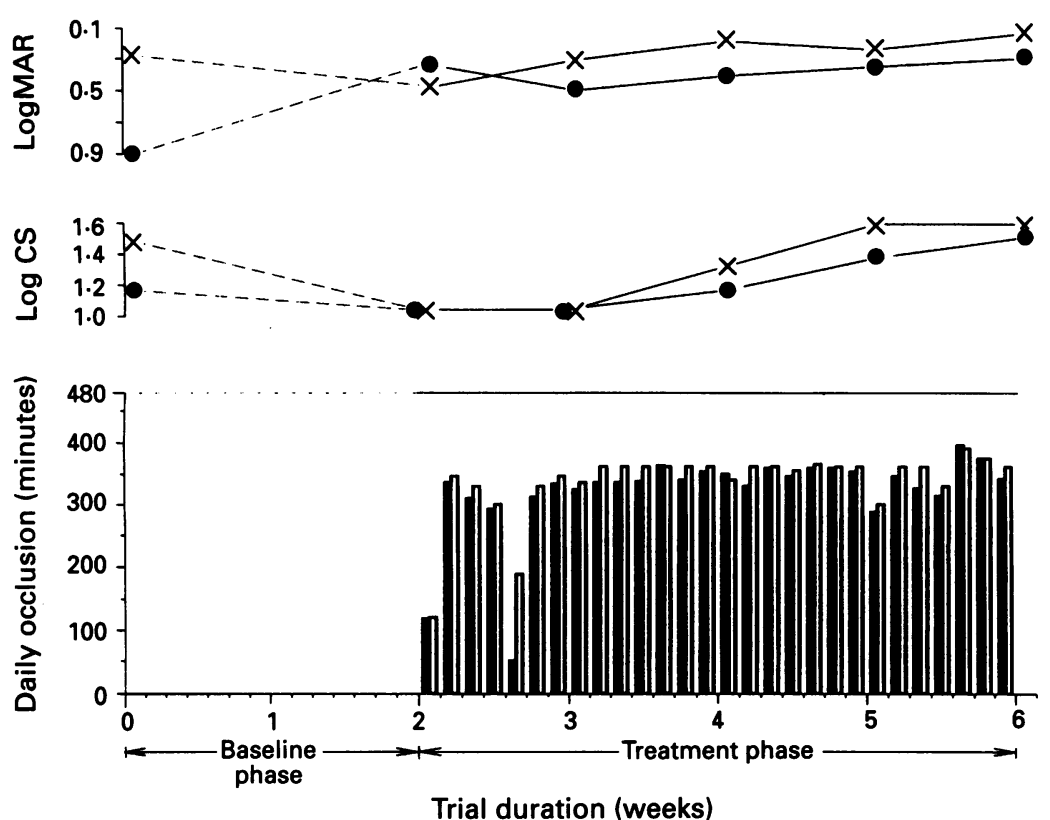

Figure 4 Example of compliance monitoring of an 8 hours per day regimen (subject 3). Key as for Figure 1. against visual function. This is not before time, as around $48 \%$ and $28 \%$ of amblyopic children are prescribed respectively $200-499$ hours and more than 500 hours of this unpleasant treatment. ${ }^{7}$ Objective monitoring has opened up new research avenues and hopefully this will enable the dose-effect relation of occlusion therapy in the various types of amblyopia to be investigated objectively, and with a precision not previously possible. The design of effective therapeutic regimens should follow which will ensure that children receive enough occlusion, but no more.

We thank the mother of illustrative case 3 for permission to present Figure 1. We acknowledge Roger Bunce, Wolfson Applied Technology Laboratories, University of Birmingham, for help with development of the ODM and Graham Hollins, with development of the ODM and Graham Hollins,
Birmingham Maternity Hospital for help with ODM modifications.

1 Von Noorden GK. Binocular vision and ocular motility: theory and management of strabismus. 4th edn. St Louis: CV Mosby, 1990.

2 Stewart-Brown SL, Haslum MN, Howlett B. Preschool vision screening: a service in need of rationalisation vision screening: a service in

3 Flynn JT, Cassady JC. Current trends in amblyopia therapy. Ophthalmology 1978; 85: 428-50.

4 Scott WE, Dickey CF. Stability of visual acuity in amblyopic patients after visual maturity. Graefes Arch Clin Exp Ophthalmol 1988; 226: 154-7.

5 Unwin B. The treatment of amblyopia - a historical review. Br Orthopt f 1991; 48: 28-31.

parents were aware that occlusion was being investigated. Legibility sometimes introduced interpretative difficulties. The primary purpose of the diary was as an equipment check and to highlight possible misunderstandings resulting from ODM use, rather than as a compliance monitor. One might conclude that since records were almost as accurate as the ODM, the latter was superfluous, but obviously diary accuracy could be influenced by ODM usage. ${ }^{18}$ Furthermore, the ODM provides us with accurate information on the integrity of occlusor-skin contact during occlusion periods, revealing episodes of 'peeping' - something we could not expect to gain from a diary. We also recognise that the parents involved in this research might not be fully representative of routine clinical practice, being keen to participate.

Even these preliminary studies have provided clinical insight into occlusion therapy hitherto unavailable. For subject 1, compliance would certainly have been doubted, but given the excellent objectively determined results we can rule out non-compliance as an explanation for poor outcome. Compliance is generally only questioned in outcome failure, and success in subject 2 might therefore have been incorrectly attributed to strict adherence to the prescribed regimen. This raises the issue that perhaps some patching regimens prescribed are excessive.

Compliance can now be evaluated accurately thus permitting precise titration of occlusion
6 Hiscox F, Strong N, Thompson JR, Minshull C, Woodruff G. Occlusion for amblyopia: a comprehensive survey of outcome. Eye 1992; 6: 300-4.

7 Duke-Elder S, Wybar K. Abnormal ocular motility. In: Duke-Elder $\mathbf{S}$, Wybar $\mathbf{K}$, eds. Ocular motility and strabismus. System of ophthalmology. Vol VI. London: strabismus. System of

8 Watson PG, Sanac AS, Pickering MS. A comparison of various methods of treatment of amblyopia: a block study. Trans Ophthalmol Soc UK 1985; 104: 319-28.

9 Eggers HM. Amblyopia. In: Diamond GH, Eggers HM eds. Strabismus and pediatric ophthalmology. In: Podos SM ed. Textbook of Ophthalmology. St Louis: Mosby, 1993 13.2-13.14.

10 Day S. Normal and abnormal visual development. In: Taylor D, ed. Pediatric ophthalmology. Oxford: Blackwell Scientific Publications, 1990: 7-20.

11 Wright EC. Non-compliance - or how many aunts has Matilda? Lancet 1993; 342: 909-13.

12 Oliver M, Neumann R, Chaimovitch Y, Gotesman N. Shimshoni $M$. Compliance and results of treatment for amblyopia in children more than 8 years old. $A m f$ Ophthalmol 1986; 102: 340-5.

13 Lithander J, Sjostrand J. Anisometropic and strabismic amblyopia in the age group 2 years and above: a prospective study of the results of treatment. $\mathrm{Br} \mathcal{F}$ Ophthalmol 1991; 75: 111-6.

14 Nucci P, Alfarano $R$, Piantanida A, Brancato $R$. Compliance in antiamblyopia occlusion therapy. Acta Ophthalmol 1992; 70: 128-31.

15 Fells P. Amblyopia - an historical perspective. Eye 1990; 4: 775-86.

16 Weckert H. Neue Wege zur Bekämpfung der Schiefamblyopie. Zentralblatt für die gesamte Ophthalmologie und ihre grenzgebiete 1932; 27: 239.

17 Fulton AB, Mayer DL. Esotropic children with amblyopia: effects of patching on acuity. Graefes Arch Clin Exp Ophthalmol 1988; 226: 309-12.

18 Claydon BE, Efron N. Non-compliance in general health care. Ophthalm Physiol Optom 1944; 14: 257-64.

19 Smith $R$. Where is the wisdom...? BMF 1991; 303: 798-9.

20 Fielder AR, Auld R, Irwin M, Cocker KD, Jones $H S$, Moseley MJ. Compliance monitoring in amblyopia Moseley MJ. Compliance m.

21 Aldridge $D$. Single-case research designs for the clinician. flridge D. Single-case research desi

22 Moseley MJ, Jones HS. Visual acuity: calculating appropriate averages. Acta Ophthalmol 1993; 71: 296-300. 